\title{
Pollination biology of Impatiens cuspidata Wight and Arn. (Balsaminaceae), a rare and endemic balsam of the Western Ghats, India
}

\author{
A.K. Sreekala ${ }^{1}$, A.G. Pandurangan ${ }^{2}$, R. Ramasubbu ${ }^{3} \&$ Shrishail K. Kulloli $^{4}$ \\ 1,2 Scientist, Plant Systematics and Evolutionary Science Division, Tropical Botanic Garden and Research Institute \\ Pacha-Palode, Thiruvananthapuram, Kerala 695562, India \\ ${ }^{3}$ In charge, Horticulture Section, CSIR Madras Complex, Tarmani, Chennai, Tamil Nadu 600113, India \\ ${ }^{4}$ Field Officer, Spice Board, Developmental wing, Somawarpet, Karnataka, India \\ Email: ${ }^{1}$ ak.sreekala@gmail.com (corresponding author), ${ }^{2}$ agpandurangan@mail.com, ${ }^{3}$ racprabha@yahoo.com, ${ }^{4}$ sreekulloli@gmail.com
}

Date of publication (online): 26 June 2011 Date of publication (print): 26 June 2011 ISSN 0974-7907 (online) | 0974-7893 (print)

Editor: K.R. Sasidharan

Manuscript details:

Ms \# 02385

Received 12 January 2010

Final revised received 11 May 2011

Finally accepted 28 May 2011

Citation: Sreekala, A.K., A.G. Pandurangan, R. Ramasubbu \& S.K. Kulloli (2011). Pollination biology of Impatiens cuspidata Wight and Arn. (Balsaminaceae), a rare and endemic balsam of the Western Ghats, India. Journal of Threatened Taxa 3(6): 1818-1825.

Copyright: @A.K. Sreekala, A.G. Pandurangan R. Ramasubbu \& Shrishail K. Kulloli 2011. Creative Commons Attribution 3.0 Unported License. JoTT allows unrestricted use of this article in any medium for non-profit purposes, reproduction and distribution by providing adequate credit to the authors and the source of publication

Author Details: see end of this article.

Author contributions: AKS designed the work corrected the manuscript and guided during the study. AGP coordinated the work, raised the funds, provided necessary comments and guidance for the field work and manuscript preparation. RR carried out the field and laboratory work and consolidated the results and prepared the manuscript. SKK assisted during the filed work and manuscript preparation

Acknowledgement: The financial support received through a Major Research Project [No SP/SO/PS-09/2003 dated 17-03-2005] from the Department of Science Technology, New Delhi is gratefully acknowledged. We thank Director TBGRI for extending a necessary logistic support during the project. Thanks are due to R. Thulashidharan for help during field collection.

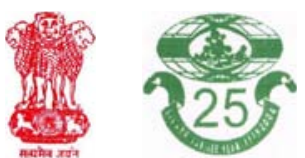

(口)

OPEN ACCESS | FREE DOWNLOAD
Abstract: The pollination biology of Impatiens cuspidata, a rare and endemic balsam from the Western Ghats, has been studied with special reference to phenology, pollination, pollen-pistil interactions, breeding experiments and stigma receptivity. It flowers at night between 2330 and 0430 hr; flowering days extend up to 160 days in a year. The anther dehisced one day before anthesis, which confirmed the protandrous condition of the flower. Pollen-ovule ratio was calculated as 1729:1. Hawk moths, honeybees, flies and butterflies are the major pollinators of Impatiens cuspidata. Pollen grains are oval, having an average diameter of $28.24 \mu \mathrm{m}$. Pollen viability by FCR test confirmed that $82 \%$ pollen grains are viable on the day of anthesis. Best pollen germination along with $1636 \mu \mathrm{m}$ tube development was achieved in Brewbakers medium. Stigma was more receptive (up to $80 \%$ ) on the first day of flower opening. It chiefly reproduced by means of cross pollination, where the fruit set was only $40 \%$, but artificial cross-pollination through xenogamy enhanced fruit set up to $80 \%$. The plant is an obligate out-crosser and self incompatible, as confirmed by various hand pollination experiments. Seed germination in natural and controlled conditions was only $20 \%$. Its dependence on a specialized habitat, bottlenecks in sexual reproduction, low percentage of seed germination and other abiotic factors could be reasons for its limited distribution and endemism.

Keywords: Impatiens cuspidata, phenology, pollination biology, stigma receptivity, xenogamy.

Tamil Abstact: அரிய மற்றும் குறிப்பிட்ட இடச்சூழல்களைக் கொண்ட மேற்குத்தொடா்ச்சி மலையின் இம்பேஸியன்ஸ் கஸ்ப்பிடேட்டா என்ற பால்ஸம் வகைத்தாவரத்தின் மகரந்தச் சேர்க்கை உயிாியல் பற்றி விரிவான ஆய்வு செய்யப்பட்டது மற்றும் இந்த ஆய்வில் முழு உடற்கூறு வளர்ச்சி, மகரந்தச்சேர்க்கை, மகரந்தம்- சூல் இடைவினை, மற்றும் இனப்பெருக்க அமைப்பு ஆகியவை பற்றி மிகுந்த கவனம் எடுத்துக்கொள்ளப்பட்டது. இதன் மலர் விரியும் நேரம் இரவு 10.00 மணி முதல் விடியற்காலை 04.30 வரை எனக்கணக்கிடப்பட்டது மற்றும் வருடத்திற்கு 160 நாட்கள் பூக்கும் என அறியப்பட்டது. மகரந்தம் பூ விரிவதற்கு ஒரு நாளுக்கு முன்பாகவே வெடிக்கத்தொடங்குகிறது இது முன்ஆண்மை முதிர்ச்சி அடைதலைக் காட்டுகிறது. ஒரு மலரில் மகரந்த தூள்-சூல் வித்து, 1729 க்கு ஒன்று என்ற விகிதத்தில் அமைந்துள்ளது. பருந்துப்பூச்சி, தேனீக்கள், ஈக்கள் மற்றும் வண்ணத்துப்பூச்சிகள் முக்கிய மகரந்தச்சோ்க்கைக் காரணிகளாகும். மகரந்தத் தூள் வட்ட வடிவமுடையதாகவும் சராசாியாக அதன் விட்டம் 28.24 நுண்ணளவு ஆகும். பூ விரிந்த நிலையில் மகரந்த தூள்களின் வாழுமை 80 சதவீதம் என்று எஃப்.சி.ஆர் என்ற ஆய்வு மூலம் உறுதி செய்யப்பட்டது. சிறந்த மகரந்தத் தூள் முளைப்புத்திறன் (82 சதவீதம்) மற்றும் மகரந்த குழாயின் நீளம் 1639 நுண்ணளவு என்பது புரூபெக்கர் எனப்படும் வளரளம் மூலம் பெறப்பட்டது. பூ விரிந்த முதல் நாள், சூல் முடியின் கருவுறும் திறன் 80 சதவீதம் வரைக் கணக்கிடப்பட்டது. இது அயல் மகரந்தச்சோ்க்கை மூலம் அதிகமாக இனப்பெருக்கம் செய்கிறது, இதன் மூலம் உண்டாகும் கனி உருவாதல் சதவீதம் 40 ஆகும் ஆனால் செயற்கை முறை அயல் மகரந்தச்சேர்க்கை (ஸீனொகேமீ) இதன் கனி உருவாதல் சதவீ தத்தை 80 ஆக உயா்த்தியுள்ளது. இந்தத் தாவரமானது உறுதி செய்யப்பட்ட அயல்மகரந்தச்சேர்க்கையாளர் என்பதும் தன்மகரந்தச்சேர்க்கைவிரும்பாதது என்பதும்பல்வேறு செயற்கைஅயல்ம கரந்தச்சேர்க்கைஆய்வுகளின் மூலம்றுதி செய்யப்பட்டது. இதன் விதை முளைப்புத்திறன் வெறும் 20 சதவீ தம்மட்டுமே. இதன் தனிப்பட்ட இடச்சூழல்வாழுமை, பல்வேறு இனப்பெருக்கத் தடைகள், குறைந்த விதை முளைப்புத்திறன் மற்றும்பல்வேறு காரணிகள்தான் இதன் குறைவான இடச்சூழல்பகிர்மானத்திற்கும்குறிப்பிட்ட இடச்சூழி ஆவதற்கும்காரணம்ஆகின்றன. 


\section{INTRODUCTION}

Pollination biology provides a framework to test a diverse array of paradigms in several subdisciplines of biology (Bawa et al. 1993). It also plays a critical role in the formation of seed and fruits. There are very few attempts made on pollination biology of Impatiens except by Rust (1977, 1979), Tian et al. (2004), Sreekala et al. (2007, 2008). A comprehensive study on pollination biology of Impatiens was fundamental to investigations into its reproductive syndrome, systematics and evolutionary biology.

The family Balsaminaceae comprises two genera; Hydrocera Blume and Impatiens Linn., which are commonly known as balsams, jewel weed, snap weed or touch-me-not (Willis 1973). The genus Impatiens is supposed to be one of the largest group among the flowering plants (Sreekala et al. 2007), comprising more than 1000 species (Grey-Wilson 1980; Clifton 2000; Pieter et al. 2006). It is essentially sub-cosmopolitan species and mainly distributed in montane areas in the Old World tropics and subtropics (Grey-Wilson 1980; Yuan et al. 2004), but several species occur in temperate Eurasia and North America (Pieter et al. 2006). Native species are absent from the South America and Australia. Impatiens is phytogeographically a unique genus, which has its greatest development in the Indian region and is found chiefly in moist sub-temperate areas.

In India, the concentration of Impatiens species is remarkably local and occurs in three major centers of diversity including the Himalaya in the north, Western Ghats in the south and parts of northeastern states. Although the altitude in southern India is lower compared with the Himalaya, conditions are favourable for the growth of Impatiens since the region gets rainfall from both southwest and northeast monsoons. There is no doubt that in respect of Impatiens, the Western Ghats are the second richest area in the Indian subcontinent and perhaps in the World (Bhaskar 1981). The genus contains over 206 species in India (Vivekananthan et al. 1997; Vishwanathan \& Manikandan 2003; Bhasker 2006), half of which occur in southern India and more than 86 are endemic to the Western Ghats (Nair 1991). Due to their restricted distribution, nearly 30 species of Impatiens are already threatened with uncertain future (Vajravelu \& Daniel 1983; Pandurangan \& Pushpangadan 1997; Sreekala et al. 2008).

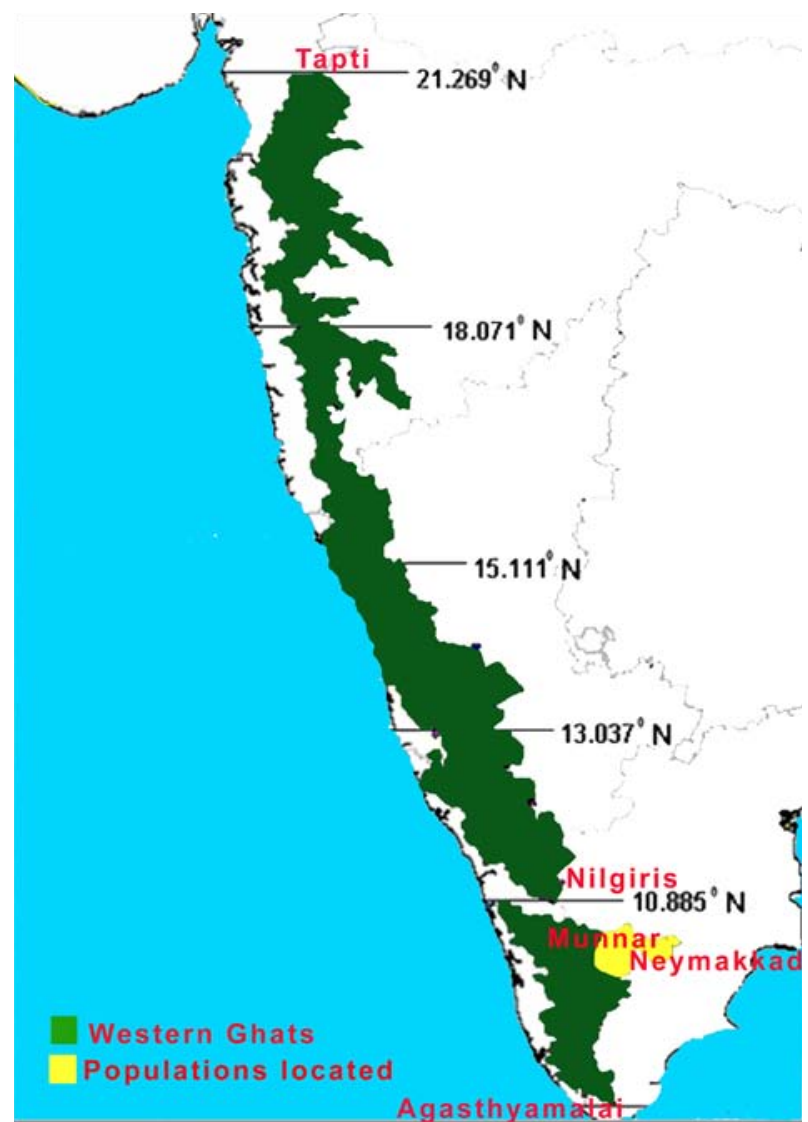

Figure 1. The study area in the Western Ghats

Though the ideal climatic conditions prevailing in the Western Ghats region provide suitable habitat for the balsams, their populations are rapidly declining due to various biotic and abiotic factors. A comprehensive study on pollination biology of endemic balsams has not been made so far due to their habitat specificity, island biogeography, delicate nature and explosive fruits. Against this background, one such wild ornamental balsam namely Impatiens cuspidata has been selected for the present investigation on pollination biology encompassing phenology, pollination, pollinator behaviour, pollen viability, stigma receptivity and breeding behaviour to find out possible reasons for its limited distribution in the Western Ghats.

\section{MATERIALS AND METHODS}

Impatiens cuspidata naturally occurs in evergreen, sholas and montane grasslands of southern Western Ghats (Fig. 1). The study was conducted on Impatiens cuspidata in natural conditions, which was located from 
Neymakkad gap of Munnar, Idukki District, Kerala, India. The area is located between $9^{\circ} 15^{\prime}-10^{\circ} 21^{\prime} \mathrm{N}$ and $76^{\circ} 15^{\prime}-77^{\circ} 25^{\prime} \mathrm{E}$. Impatiens cuspidata is a shrub and may attain a height of $1.5 \mathrm{~m}$ or even more in dense shola forests at an altitude of 1500-2000 m (Image 1a). This species is found to be associated with Impatiens leschenaultii, I. coelotropis, I. henslowiana, Rubus sp. Alternanthera sp. and Rhodomytrus sp. The area experiences rainfall from both southwest and northeast monsoons and receives an average rainfall of $3000 \mathrm{~mm}$ except in Anaimudi where some times rainfall exceeds $7000 \mathrm{~mm}$. The region harbours vegetation types such as moist deciduous, semievergreen, evergreen, shola and montane grasslands. The study was conducted during March 2005 and December 2007. Five populations were selected for the present investigation in the natural condition. Twenty healthy plants were selected from each population and observations were made on a day-to-day basis in natural habitats on flowering phenology, which include season, habit, development, anthesis etc. Floral morphology was also studied with the help of hand lens and dissection microscope. Fifty flower buds were selected from different populations and observations were made between 2330 to 0830 hrs to study the time of flower opening (anthesis) and anther dehiscence. The number of pollen grains contained in each anther was determined by the method suggested by Cruden (1977) to determine P/O ratio of the candidate species.

Pollen fertility was assessed by acetocarmine and glycerin staining technique. The stained pollen grains were treated as fertile and unstained pollens were counted as sterile. Pollen viability was checked by FCR (fluorochromatic reaction) test using fluorescein diacetate (FDA). To study the pollen germination in vitro, pollen grains were incubated in sucrose medium of different concentrations $(2,5,10,15, \&$ $20 \%$ ) and Brewbakers medium (Brewbaker \& Kwack 1963 ) containing $2 \%$ sucrose for two hours. After two hours the percentage of pollen germination and tube elongation was noticed. Stigma receptivity was studied visually with the help of hand lens and by hydrogen peroxide $\left(\mathrm{H}_{2} \mathrm{O}_{2}\right)$ test according to the method of Scribailo \& Posluszny (1984). In vivo pollen germination was checked by using aniline blue (Aldrich chemical 86.102-2) florescence microscopic method as designed by Shivanna \& Rangaswamy (1992). The preparations were observed under the fluorescent microscope (Lieca DME Germany). Percentages of pollen germination in the stigmatic surface and average tube length were calculated.

Continuous observations during July 2005 to March 2006 were made on behaviour of different pollinators. The pollinators were collected and identified with the help of experts from Kerala Agricultural University and KFRI (Kerala Forest Research Institute). The foraging period and the type of food collected by different visitors on daily basis were recorded by close observations. Different pollination systems such as autogamous self pollination (B), emasculation and hand crossing $(\mathrm{S})$, emasculation and hand out crossing $(\mathrm{O})$ and natural pollination $(\mathrm{N})$ were tested in the field. Treatment ' $S$ ' was conducted to examine geitonogamy through artificial pollination using pollens from different flowers of the same plant. Treatment ' $\mathrm{O}$ ' was conducted to examine xenogamy through artificial cross pollination by using pollens from flowers of different plants but within the populations. Twentyfive healthy flowers for each treatment from each population were chosen randomly and observed for fruit set. The developed fruits and seeds were collected and their numbers were recorded. In addition, the weight of each seed was also measured and recorded. Twenty mature capsules were selected randomly from five populations in the field for this experiment. The distance to which seeds ejected from the capsules were measured. In the laboratory 240 seeds, from capsules of each plant were taken and soaked overnight to

Table 1. Floral biology of Impatiens cuspidata

\begin{tabular}{|l|l|}
\hline Floral characters & Observations \\
\hline Flowering period & August-December \\
\hline Flower opening time & $1130-0430 \mathrm{hrs}$ \\
\hline Flower colour & Light pink \\
\hline No. of anthers/flower & 5 \\
\hline Anther dehiscence time & One day before anthesis \\
\hline Mean no. of pollen grains/flower & 34,571 \\
\hline Pollen size & $28.24 \mu \mathrm{m}$ \\
\hline Stigma type & Wet and non-papillate type \\
\hline Stigma receptivity & $0800-0230 \mathrm{hr}$ \\
\hline Mean no. of ovules/flower & 20 \\
\hline Fruit setting in natural condition & $40 \%$ \\
\hline Pollen fertility & $80 \%$ \\
\hline Pollen viability & $82 \%$ \\
\hline Pollen germination $\%$ & $96 \%$ \\
\hline
\end{tabular}



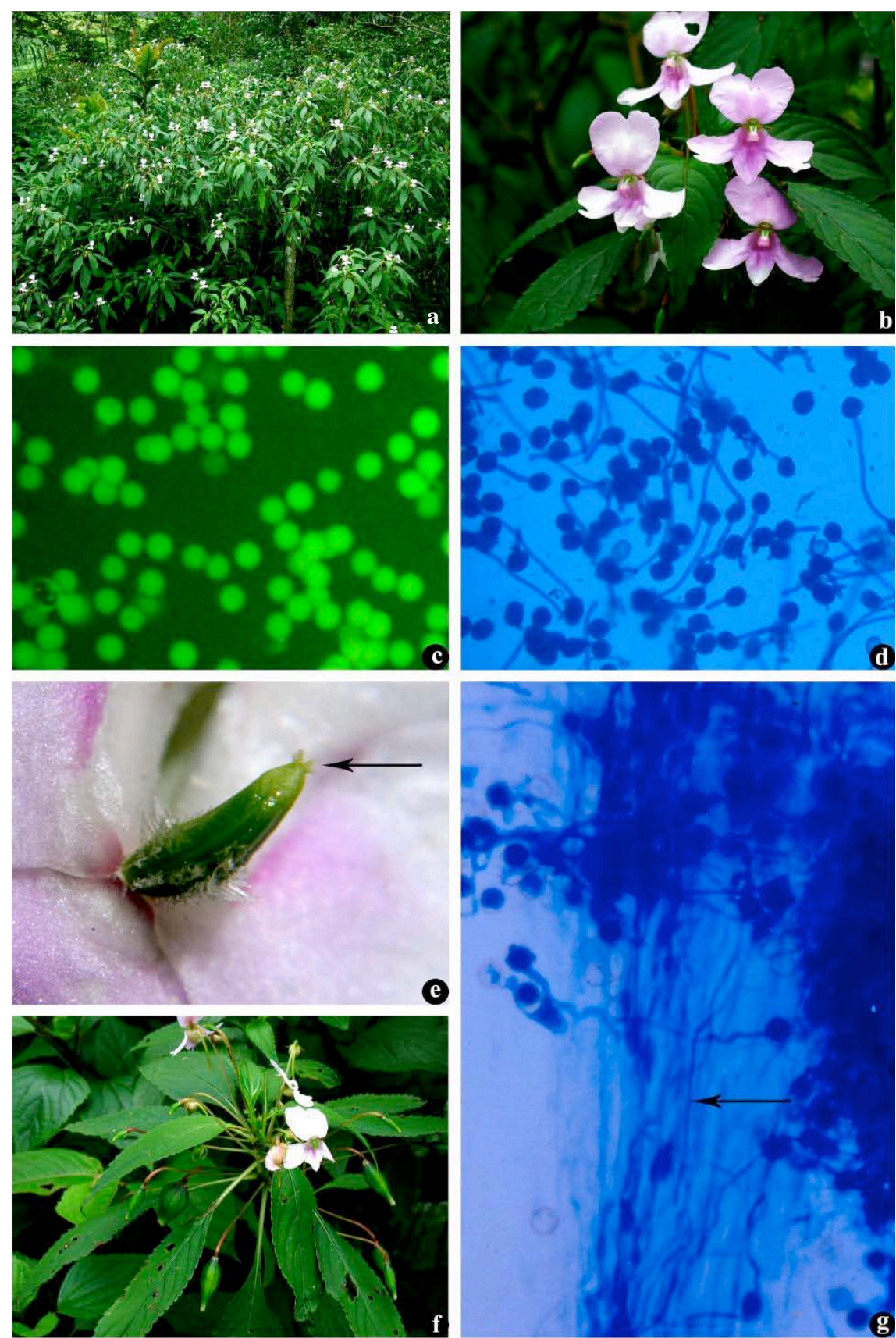

Image 1. Pollination biology of Impatiens cuspidata

a - population of I. cuspidata; b - full bloom flowers; $c$ - pollen viability by FCR test; $d$ - in vitro pollen germination by Brewbakers medium;

e - receptive stigma with stigmatic lobes; $f$ - in vivo pollen germination; $f$ - fruit set in natural habitat

soften the seed coat. The soaked seeds were then placed on germination paper and incubated at $25^{\circ} \mathrm{C}$ and germination percentage was recorded.

\section{RESULTS}

The flowers of I. cuspidata are light pink in colour (Image 1b) born at apical cyme in pair or solitary and spur is straight, oblong and glabrous. Capsules are ellipsoid and cuspidate in nature; contains 14-16 seeds in each capsule. The plant starts flowering in the month of August and it extends up to December with peak flowering during October. The flower buds take 6-11 days from initiation to full bloom. The flowering period is extended up to 160 days in a year and the average life span of the individual flower is $2-3$ days. The flowers bloom in the night between 2330 and 0430 $\mathrm{hr}$, confirming their nocturnal nature. Anther dehisced one day before flower opening which confirmed the protandrous condition of the flowers (Table 1). The mean number of pollen grains per flower was found to 
Table 2. In vivo pollen germination of Impatiens cuspidata

\begin{tabular}{|l|c|c|c|c|}
\hline Treatments & \multicolumn{4}{|c|}{ Period after flower opening (anthesis) } \\
\hline & I day & II day & III day & Drooping stage \\
\hline Total no. of stigmas observed & 10 & 10 & 10 & 10 \\
\hline No. of stigmas showing germination & 8 & 4 & 2 & 0 \\
\hline$\%$ of stigma receptivity & 80 & 40 & 20 & 0 \\
\hline Total no. of pollen retained on stigma & 28,520 & 3,430 & 165 & 11 \\
\hline Mean no. of germinated pollen & 11,430 & 865 & 14 & -- \\
\hline$\%$ of pollen germination & 62 & 23 & 6 & -- \\
\hline Pollen tube length $(\mu \mathrm{m})$ & 917 & 136 & 46 & -- \\
\hline
\end{tabular}

be 34,571 and the mean number of ovules was twenty. Therefore the pollen ovule ratio was calculated as 1729: 1 (Table 1).

Pollen grains are oval and have an average size of $28.24 \mu \mathrm{m}$ in diameter. The acetocarmine staining technique revealed that $80 \%$ of the pollen grains were fertile. Pollen viability by FCR test confirmed that $82 \%$ pollen grains were viable on the day of anthesis (Image 1c) and gradually reduced after second day of anthesis. In vitro pollen germination studies by using different concentration of sucrose and Brewbakers medium revealed that pollen grains were viable only up to the minimal period. Effect of sucrose on in vitro pollen germination studies revealed that $68 \%$ of pollen grains germinated and produced $1003 \mu \mathrm{m}$ tube in $5 \%$ sucrose medium. Best pollen germination (96\%) along with $1636 \mu \mathrm{m}$ tube development was achieved in Brewbakers medium after four hours of incubation (Image 1d).

Receptivity of stigma is a critical factor for successful completion of post pollination events. The stigma is wet and non-papillate type (Heslop-Harrison $\&$ Shivanna 1977). Stigmas were more receptive up to $80 \%$ on the first day of flower opening (Table $2 \&$ Image 1e); by showing $62 \%$ in vivo germinating pollens along with $917 \mu \mathrm{m}$ long pollen tube on the stigmatic surface (Table $2 \&$ Image 1g). Subsequently, the receptivity percentages and in vivo germinating pollens were decreased on successive days after blooming. In I. cuspidata, pollen grains were well adhered on the stigmatic surface due to sticky nature and presence of pollen threads. Pollen tubes penetrate the stigmatic region and reached up to the ovary and fertilized the ovules. The fertilized ovules developed into seeds with only $20 \%$ germinability.

When I. cuspidata flowers bloom in the night
Table 3. Pollinators and their characteristics in Impatiens cuspidata

\begin{tabular}{|l|l|l|l|}
\hline Visitors & $\begin{array}{l}\text { Visiting } \\
\text { time }\end{array}$ & $\begin{array}{l}\text { Foraging } \\
\text { nature }\end{array}$ & $\begin{array}{l}\text { Foraging } \\
\text { hours }\end{array}$ \\
\hline Apis cerana & Day & Nectar \& pollen & $0700-0400 \mathrm{hr}$ \\
\hline Trigona sp. & Day & Nectar \& pollen & $0730-0230 \mathrm{hr}$ \\
\hline $\begin{array}{l}\text { Macroglossum } \\
\text { variegatum }\end{array}$ & Night & Nectar & $1140-0330 \mathrm{hr}$ \\
\hline M. corythus & Night & Nectar & $1230-0300 \mathrm{hr}$ \\
\hline Butterflies & Day & Nectar & $0630-0500 \mathrm{hr}$ \\
\hline Flies & Day & Nectar & $0730-0330 \mathrm{hr}$ \\
\hline
\end{tabular}

between 2330 and $0430 \mathrm{hr}$, two species of hawk moths: Macroglossum corythus and M. variegatum were found to forage actively. They helped in pollination by their rapid and frequent visit to flowers for nectar collection. Pollen loads were found on the head and long proboscis of hawk moths, which transferred pollen grains from one flower to another from the same plant or another plant and thus favoring geitonogamy or xenogamy. The foraging periods of honeybees were mainly during daytime from 0700 to $1600 \mathrm{hr}$. Honey Bees visited many flowers and spent an average of 2-4 seconds in each flower, whereas hawk moths spent slightly long duration from 3-6 seconds in each flower. Bees (Apis cerana indica \& Trigona sp.) were found to be the most abundant visitors and they visited more flowers than any other pollinators. Butterflies were active during daytime and in fine weather, they actively visited the flowers, spending an average of $3-5$ seconds on individual flower for nectar. However, when the weather was cloudy, butterflies were less active and in rainy days, they were completely inactive. Flies were intermittent visitors and found to be poor pollinators (Table 3). In general, hawk moths in night and honey bees in day time served as better pollinators, but they 
Table 4. Fruit set in different modes of pollination in Impatiens cuspidata

\begin{tabular}{|l|c|c|c|}
\hline Treatments & $\begin{array}{c}\text { No. of flowers } \\
\text { pollinated }\end{array}$ & $\begin{array}{c}\text { No. of } \\
\text { flowers set } \\
\text { fruit }\end{array}$ & $\begin{array}{c}\% \text { of fruit } \\
\text { set }\end{array}$ \\
\hline $\begin{array}{l}\text { Bagged (B)/ } \\
\text { autogamous-self }\end{array}$ & 25 & 0 & 0 \\
\hline $\begin{array}{l}\text { Emasculation and } \\
\text { hand crossing (S) }\end{array}$ & 25 & 14 & 56 \\
\hline $\begin{array}{l}\text { Emasculation and } \\
\text { hand out crossing (O) }\end{array}$ & 25 & 19 & 76 \\
\hline Natural pollination (N) & 25 & 10 & 40 \\
\hline
\end{tabular}

were not sufficient to pollinate all the flowers in the selected populations.

In I. cuspidata, different breeding experiments were carried out to find the reproductive capacity of the plant. In natural condition, $40 \%$ fruit set was observed (Image 1f). The fruit set was not observed in autogamous self pollination. However, 56\% fruit set was observed in geitonogamy and $76 \%$ in xenogamy (Table 4; Fig. 2). Breeding experiments like geitonogamy and xenogamy produced more fruits and seeds than the natural pollination. The average weight of the individual seeds produced by the treatments of natural, geitonogamy and xenogamy were 1.5, 1.7, $2.0 \mathrm{mg}$ respectively which indicated that the seeds produced by artificial pollination were more healthy and viable than natural system because of combination of gametes from different flowers or plants. The fruit development took 25-30 days for attaining maturity after fertilization. As capsules mature, the fruit wall ruptured and the seeds were ejected up to 0.60-1.25 $\mathrm{m}$ away from the mother plant and this is the only way of dissemination of seeds in the present taxa. Seeds germinate after dehiscing from the capsule in a favorable place but very few of them established into seedlings in the natural condition. But seeds developed through xenogamy produced more seedlings. In the laboratory condition, the germination of seeds obtained through natural pollination was $20 \%$.

\section{DISCUSSION}

The members of Balsaminaceae have their greatest development in the Indian region and are remarkably endemic. Endemism gives us clue that they are in restricted distribution, either due to their reproductive syndrome or by anthropogenic pressures. Knowledge on phenology and floral morphology are essential for

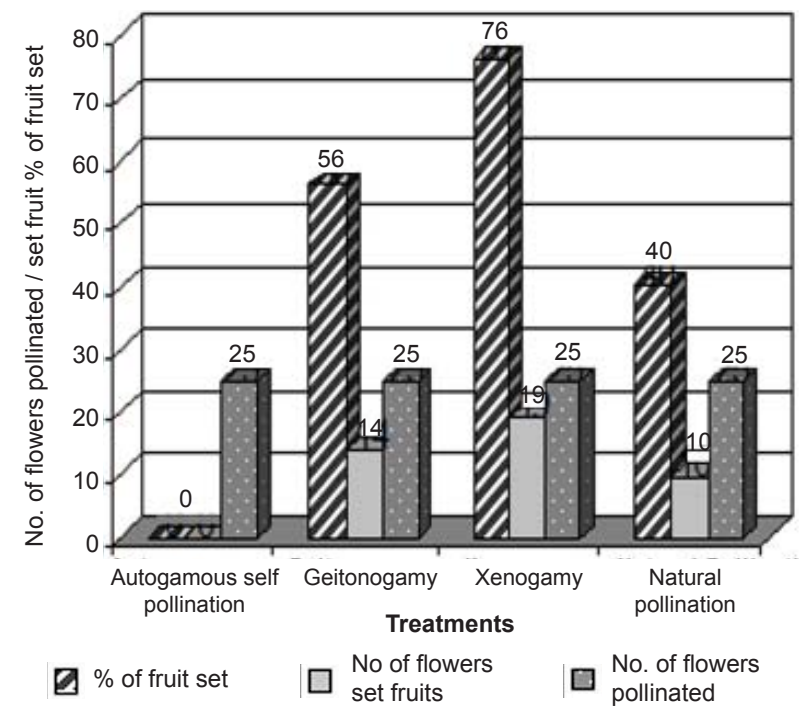

Figure 2. Fruit set in different modes of pollination systems in Impatiens cuspidata

conducting studies on breeding systems particularly on pollination syndrome if any. Impatiens cuspidata starts flowering in the month of August and continued up to December and reached a peak during October and anthesis commenced between 2330 and $0430 \mathrm{hr}$ on the next day. About $62 \%$ of Impatiens species in the Western Ghats flower during July-December, 16\% during April-June and 15\% during January-March. Interestingly $18 \%$ of the balsams flower throughout the year if conditions are favorable (Rajalal et al. 1996). Bhaskar \& Razi (1974) had reported that majority of the wild balsams grown in the high altitude areas are night blooming and have a wide range of timing with regard to pollen germination. The anther dehisced one day before anthesis, which in turn confirmed their protandrous condition. This observation was similar to that of I. platypetala, I. korthalsii, and I. eubotrya in Sumatra (Kato et al. 1991).

In I. cuspidata, pollen viability is highest on the day of anthesis and then gradually decreased on successive days after anthesis. This observation is similar to that of $I$. reptans in China (Tian et al. 2004). In vitro pollen germination test indicated that highest percentage of pollen germination and tube elongation was observed in Brewbakers medium. Sucrose acts as a nutritive material for pollen germination (Johri \& Vasil 1961) and it helps in maintaining osmotic balance between the germination media and pollen cytoplasm (Mukerjee \& Das 1964). Germination percentages were significantly low in higher concentration of sucrose medium. 
According to Shivanna \& Johri (1985), the optimum concentration of sucrose varies from species to species. In the present investigation, Brewbakers medium is the most suitable for pollen germination in I. cuspidata. Besides the medium contain carbohydrates, boron and calcium which are other important substances required for pollen germination and tube growth (Brewbaker \& Kwack 1963). Pollen germination and subsequent post pollination events depend upon the receptivity of the stigma, its nature and compatibility.

It is well known that the flowers of Impatiens have enormous diversity and different pollinators. Impatiens cuspidata is pollinated by honeybees, hawk moths and butterflies. In different climatic regions, species of pollinators vary. In sub tropical regions of Africa the Impatiens species are pollinated by humming birds as well as by insects. In temperate zones, pollinators are bumblebees and humming birds (Rust 1977, 1979; Heinrich 1979; Kato et al. 1989). In I. cuspidata, bees (Apis cerana indica and Trigona sp.) are the most important pollinators and visited more flowers than any other pollinators during day time for nectar and pollen gathering. There is a strong relationship between the weather and foraging activity of pollinators. When the weather is fine, butterflies are more active and spend on an average 2-6 seconds on a flower at each visit. But when the weather is cloudy and rainy, the butterflies and hawk moths are less active. The present investigation agrees with the findings on I. coelotropis (Sreekala et al. 2008).

In I. cuspidata, $40 \%$ fruit set was observed in natural pollination. However, artificial cross pollination (geitonogamy and xenogamy) enhanced the fruit set rate up to $76 \%$. Pollination experiments demonstrated that, artificial cross-pollination enhanced the rate of fruit and seed set in I. cuspidata. The balsams are highly evolved members among the order Geranials as evident from their marked zygomorphic flowers and nectariferous spur. The arrangement of stamens, pistil and spur are markedly adapted for cross pollination in Impatiens (Bhaskar \& Razi 1974) and hence most of the species of Impatiens reproduce by cross pollination (Schmitt \& Gamble 1990; Lu 2000, 2002).

Stigma receptivity is a critical factor for successful completion of post pollination events. Usually it is highest soon after anthesis but it varies from species to species, depending upon the temperature and humidity (Shivanna \& Johri 1989). But in $I$. cuspidata, stigmas remained receptive only after the shedding of androecium and gradually increased for 8-14 hr. The receptivity ends after 14 hours but at the same time its pollen viability reduced drastically. The adhesion of pollens on the stigma is a primary requirement for successful pollination. After landing on the stigmatic surface, pollen grains are subjected to hydration and then pollen wall proteins are released on to the stigmatic surface (Heslop-Harrison et al. 1975). In I. cuspidata, pollen grains are well adhered on the stigmatic surface. Pollen tubes penetrate the stigmatic surface and reached up to the ovary and successfully fertilize the ovules. The fertilized ovules developed into seeds. The percentage of seed set was only $60 \%$.

Seeds are not dormant and they germinate immediately. Experimental results also substantiate the same. Very few germinated seeds were established into seedlings and remaining perished. This may be due to insufficient flow of nutrients into seeds. Therefore the study suggests that, absence of dormancy, protandry, self-incompatibility, pollinator limitation and perishing of considerable percentage of seedlings prior to establishment in combination with other abiotic traits are contributing factors for regulation of population size of $I$. cuspidata in its natural condition.

\section{REFERENCES}

Bawa, K.S., K.N. Ganeshaiah \& R. Umashankar (1993). Editors note. Current Science 65: 191-192.

Bhaskar, V. \& B.A. Razi (1974) Nocturnal pollen germination in Impatiens (Balsaminaceae). Current Science 43(19): 626628.

Bhaskar, V. (1981). The genus Impatiens L. in south India: endemism and affinities. Indian Forester 107(6): 368-378.

Bhaskar, V. (2006). Impatiens clavata Bhaskar sp. nov.- a new scapigerous balsam (Balsaminaceae) from Bisle Ghat Western Ghats south India. Current Science 91(9): 1138-1140.

Brewbaker, J.L. \& B.H. Kwack (1963). The essential role of calcium ion in pollen germination and pollen tube growth. American Journal Botany 50: 859-865.

Clifton, R. (2000). Geraniales Species Checklist Series: Vol 6. Geraniaceae-Bentham and Hooker tribe VIIIBalsaminaceae. Dover, The Geraniaceae Group, 396pp

Cruden, R.W. (1977). Pollen ovule ratios: a conservative indicator of breeding systems in flowering plants. Evolution 31: $32-46$.

Grey-Wilson, C. (1980). Impatiens of Africa-Morphology, Pollination and Pollinators, Ecology, Phytogeography, Hybridization, Keys and a Systematics of all African Species 
with a Note on Collecting and Cultivation. A.A. Balkema, Rotterdam, Netherlands, 235pp

Heinrich, B. (1979). Resource heterogeneity and patterns of movements in foraging bumblebees. Oecologia 40: 235245.

Heslop-Harrison, R., B. Knox, J.Y. Heslop-Herrison \& O. Mattson (1975). Pollen wall proteins: emission and role in incompatibility response, pp. 189-202. In: Deckett, J.G. \& P.A. Racey (eds.). The Biology of Male Gamete. Biological Journal of Linnaean Society 7.

Heslop-Harrison, Y. \& K.R. Shivanna (1977). The receptive surface of the angiospermic stigma. Annals of Botany 41: 1233-1258.

Johri, B.M. \& I.K. Vasil (1961). Physiology of pollen. Botanical Review 27: 325-381.

Kato, M.I., M. Itino, I. Hotta, E. Abbas \& H. Okada (1989). Flower visitors of 32 plants species in west Sumatra. Occasional papers of the Kagoshima University Research center for South Pacific No. 16: 15-31.

Kato, M.I., M. Itino, I. Hotta \& T. Inoyue (1991). Pollination of four Sumatran Impatiens species by hawk moths and bees. Tropics 1: 59-73.

Lu, Y.Q. (2000). Effect of density on mixed mating systems and reproduction in natural populations of Impatiens capensis (Balsaminaceae). International Journal of Plant Science 161: 671-681.

Lu, Y.Q. (2002). Why is cleistogamy a selected reproductive strategy in Impatiens capensis (Balsaminaceae)? Biological Journal of Linnaean Society 75: 543-553.

Mukerjee, S.K. \& P.K. Das (1964). Palynology in horticulture and plant breeding, pp. 305-326. In: Nair, P. K. K. (eds.) Recent Advances in Palynology.

Nair, N.C. (1991). Endemism on the Western Ghats with special reference to Impatiens L., pp. 93-102. In: Proceedings of the Symposium on Rare, Endangered and Endemic Plants of Western Ghats. Kerala Forest Dept. (Wildlife Wing), Thiruvanathapuram, Special Publication No: III.

Pandurangan, A.G. \& P. Pushpangadan (1997) Causes of Rarity and Extinction of Species in Plants with Reference to Western Ghats. Frontiers in Plant Science. Om Sai Graphics, Hyderabad, 381pp.

Pieter, L.C., P.G. Koen, B.T. Steven \& F.S. Erick (2006). Floral development in three species of Impatiens (Balsaminaceae). American Journal of Botany 93(1): 1-14.

Rajalal, R., A.G. Pandurangan \& P. Puspangadhan (1996). Systematic studies of Balsaminaceae in Peninsular India. Journal of Swamy Botanical Club 13: 59-62.

Rust, R.W. (1977). Pollination in Impatiens capensis and I. pallida (Balsaminaceae). Bulletin of Torrey Botanical Club 104: 361-367.

Rust, R.W. (1979). Pollination in Impatiens capensis: pollinators and nectar robbers. Journal of Kansas Entomological Society 52: 297-308.

Schmitt, J. \& S.E. Gamble (1990). The effect of distance from the parental site of offspring performance and inbreeding depression in Impatiens capensis a test of the local adaptation hypothesis. Evolution 44: 2022-2030.

Scribailo, R. W. \& U. Posluszny, (1984). The reproductive biology of Hydrocharis morsus-ranae. I. Floral biology. Canadian Journal of Botany 62: 2779-2787.

Shivanna, K.R. \& N.S. Rangaswamy (1992). Pollen Biology - A Laboratory Manual. Narosa Publishing House, New Delhi, 332pp

Shivanna, K.R. \& B.M. Johri (1989). The Angiosperm Pollen: Structure and Function. Wiley Eastern Ltd., New Delhi, 235pp.

Sreekala, A.K., R. Ramasubbu, S.K. Kulloli \& A.G. Pandurangan (2007). Pollination biology of Impatiens henslowiana Arn. (Balsaminaceae). Indian Journal Botanical Research 3(1): 165-171.

Sreekala, A.K., A.G. Pandurangan, R. Ramasubbu \& S.K. Kulloli (2008). Reproductive biology of Impatiens coelotropis Fischer. A critically endangered balsam from the southern Western Ghats. Current Science 95(3): 386-388.

Tian, J., L. Keming \& H. Guangwan (2004). Pollination ecology and pollination system of Impatiens reptans (Balsaminaceae) endemic to China. Annals of Botany 93: 167-175.

Vajravelu, E. \& P. Daniel (1983). Materials for A Catalogue of Threatened Plants of India. Botanical Survey of India, Calcutta, 265pp.

Vishwanathan, M.B. \& U. Manikandan (2003). A new species of Balsaminaceae, Impatiens tirunelvelica, from peninsular India. Bulletin Botanical Survey of India 45(1-4): 189-194.

Vivekananthan, K., N.C. Rathakrishnan, M.S. Swaminathan \& L.K. Ghara (1997). Balsaminaceae, pp. 96-229. In: Hajara, P.K., V.J. Nair \& P. Daniel (eds.). Flora of India (Malphighiaceae-Dichapatalaceae)-4 . Botanical Survey of India, Calcutta.

Willis, J.C. (1973). A Dictionary of The Flowering Plants and Ferns. Cambridge University Press, Cambridge, 591pp.

Yuan, Y.M., Y.I. Song, E.E. Geuten Koen, S. Rahelivololona, E. Wohlhauser, S. Fischer, S. Smets \& P. Kupfer (2004). Phylogeny and biogeography of Balsaminaceae inferred from ITS sequence data. Taxon 53: 391-403.

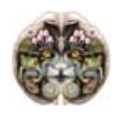

Author Details: DR. A.K. SREEKALA is currently holding a scientist position in TBGRI. She is recipient of several awards and published as many research papers in National and International journals. She presently working on reproductive biology of RET plant species from the Western Ghats. Dr. A.G. Pandurangan is scientist F \& Head, Plant Systematics and Evolutionary Science Division, Tropical Botanic Garden and Research Institute. He is the recipient of several national and international awards. $\mathrm{He}$ is selected as a member on expert committee of many organizations. $\mathrm{He}$ has more than 200 research papers in national and international Journals. He is presently working on systematics of grasses, sedges, balsams, Asclepiadaceae, floristic study of Kerala, reproductive biology of RET Balsams and. DR. R. RAmAsUBBU was working as senior research fellow in the DST funded projects and currently holding a position of Incharge, Horticulture section, CSIR-CMC, Chennai. He has published several research papers in national and international journals. MR. SHRISHAIL K. KULLOLI was also worked as senior research fellow for the same project. Now he is field officer, Spice Board, Somawarpet, Karnataka. He has published several research papers and has contributed articles on Western Ghats. 